\title{
Expanding endomorphisms of the circle revisited
}

\author{
MICHAEL SHUB AND DENNIS SULLIVAN \\ Department of Mathematics, Queens College and the Graduate School of CUNY, \\ 33 West 42nd Street, New York, NY10036 USA; IHES, 35 Rue de Chartres, \\ 91440 Bures-sur-Yvette, France
}

(Received 12 January 1984 and revised 8 October 1984)

\begin{abstract}
Two $C^{r}, r \geq 2$, expanding maps of the circle which are absolutely continuously conjugate are $C^{r}$ conjugate. Here $f$ and $g: S^{1} \rightarrow S^{1}$ are expanding if they stretch tangent vectors in some metric, and a conjugacy is an isomorphism $h: S^{1} \rightarrow S^{1}$ such that $f h=h g$.
\end{abstract}

\section{Section 0. Introduction}

The $C^{r}$ endomorphism $f: M \rightarrow M$ of the boundaryless compact differentiable manifold $M$ is expanding if in some smooth metric $f$ stretches every tangent vector. Let $S^{1}$ be the unit circle in the complex plane. Here we consider the conjugacies $h$ which may exist between two expanding endomorphisms $f$ and $g$ of $S^{1}$. We study $h: S^{1} \rightarrow S^{1}$ which is a Borel measurable bijection defined a.e., which satisfies $f h=h g$ a.e. and which is non-singular with respect to the Lebesgue measure $\lambda$

$$
\lambda(X)=0 \quad \text { iff } \quad \lambda(h X)=0 .
$$

Such an $h$ is called an absolutely continuous conjugacy between $f$ and $g$. In [6] it was proved that any two expanding maps of the same degree are topologically conjugate, that is conjugate by a homeomorphism of the circle. Generally these homeomorphisms are not Lipschitz because eigenvalues of periodic points vary. However, all these eigenvalue invariants are equal in the presence of an absolutely continuous conjugacy because of:

THEOREM 1. Let $2 \leq r \leq \omega$. If two orientation preserving expanding $C^{r}$ endomorphisms $f$ and $g$ of $S^{1}$ are absolutely continuously conjugate, then they are conjugate by $a C^{r}$ diffeomorphism.

In $\S 1$ we prove this theorem, including the proofs of some well known propositions and lemmas when their proofs fit naturally into the discussion. The proof shows that the absolutely continuous conjugacy agrees with a $C^{r}$ conjugacy except in the case when both are smoothly conjugate to $z \rightarrow z^{n}$. In $\S 2$ we discuss the real analytic case further vis à vis the Jacobian invariant of Parry and Walters. Finally we consider 
some examples among the Blaschke products, showing absolutely continuous conjugacy implies conjugacy by elements from PSl $(2, \mathbb{R})$. In $\S 2$ we mention an open problem.

\section{Section 1.}

LEMMA 1 (well known). Let $f$ be a $C^{1+\alpha}$ expanding endomorphism of $S^{1}$. There is a constant $c>0$ such that if $I \subset S^{1}$ is an interval and $f^{n}$ is injective on $I$ then

$$
\frac{1}{c}<\frac{\left|D f^{n}(y)\right|}{\left|D f^{n}(z)\right|}<c \quad \text { for any } y, z \in I .
$$

Proof. Let $y, z \in I$ and let $d(y, z, I)$ denote the distance between $y$ and $z$ measured along $I$. Thus

$$
\begin{aligned}
d\left(f^{j} y, f^{j} z, f^{j} I\right) & =d\left(\left(f^{n-j}\right)^{-1}\left(f^{n}(y)\right),\left(f^{n-j}\right)^{-1}\left(f^{n}(z)\right), f^{j} I\right) \\
& \leq \lambda^{-n+j} d\left(f^{n}(y), f^{n}(z), f^{n} I\right),
\end{aligned}
$$

where $|D f| \geq \lambda>1$ comes from the definition of an expanding endomorphism. Since $D f$ is Hölder of exponent $\alpha$ and bounded away from $0, \log |D f|$ is Hölder. So there is a constant $K$ such that

$$
\begin{aligned}
|\log | D f\left(f^{j}(y) \lambda\right)||-|\log | D f\left(f^{j}(z)\right)|| & \leq K\left(\lambda^{-n+j} d\left(f^{n}(y), f^{n}(z), f^{n} I\right)^{\alpha}\right. \\
& \leq K^{\prime}\left(\lambda^{\alpha}\right)^{-n+j}
\end{aligned}
$$

where $K^{\prime}$ is $K$ times the length of the circle. Thus

$$
\begin{aligned}
\left|\log \frac{\left|D f^{n}(y)\right|}{\left|D f^{n}(z)\right|}\right| & \leq \sum_{j=0}^{n}\left|\log \frac{\left|D f\left(f^{j}(y)\right)\right|}{\left|D f\left(f^{j}(z)\right)\right|}\right| \quad \text { by the chain rule, } \\
& \leq K^{\prime} \sum_{j=0}^{n}\left(\lambda^{-n+j}\right)^{\alpha} .
\end{aligned}
$$

Thus $c=\exp \left(K^{\prime}\left(1-\lambda^{-\alpha}\right)^{-1}\right)$ works.

COROLlary 1. If an interval I about $x$ has length less than $\left(c\left|D f^{n}(x)\right|\right)^{-1}$ then $f^{n}$ is injective on $I$.

Proof. The proof is left as an exercise.

Corollary 2. Let $x_{n}=f^{n} x, y_{n}=f^{n} y$, and $D_{n}=\left|D f^{n}(x)\right|$. If distance $(x, y) \leq\left(c D_{n}\right)^{-1}$, then distance $\left(x_{n}, y_{n}\right)$ is between $c^{-1} D_{n}$ distance $(x, y)$ and $c D_{n}$ distance $(x, y)$.

Proof. By corollary 1 the distortion lemma 1 applies to the short interval between $x$ and $y$.

COROllary 3. If $f$ is an expanding $C^{1+\alpha}$ map, and $A$ is an invariant set $(f(A) \subset A)$ of positive measure then $A$ has full measure. In particular, $f$ is ergodic.

Proof. (Well known.) Consider small intervals containing and converging to a Lebesgue density point of $\boldsymbol{A}$ which can be expanded up injectively by powers of $f$ to almost fill the circle. By the distortion lemma and the invariance of $A$ the image intervals are mostly filled by points of $A$. Thus $A$ must have full measure.

Theorem 2 (Sacksteder [5], Krzyzewski [1]). Let f be a $C^{r}$ expanding endomorphism of $M$ for $2 \leq r \leq \omega$. Then there is a normalized $C^{r-1}$ invariant measure for $f$. 
Note. $\infty-1=\infty$ and $\omega-1=\omega$ in the statement of this theorem.

Corollary 4. Let $f$ be a $C^{r}$ expanding emdomorphism of $S^{1}$ for $2 \leq r \leq \omega$. Then $f$ is $C^{r}$ conjugate to an expanding endomorphism g of $S^{1}$ which preserves Lebesgue measure.

Proof of corollary 4. Let $\mu$ be the invariant measure for $f$ given by the $C^{r-1}$ density function $\theta$ which is necessarily strictly bigger than 0 : for if $\theta$ vanishes at a point, invariance implies that $\theta$ vanishes on the backwards orbit of the point which is dense in $S$. Now let $h(x)=\int_{0}^{x} \theta d \lambda . h \mu=\lambda, h$ is a $C^{r}$ diffeomorphism and $h f h^{-1}$ preserves Lebesgue measure.

Proof of theorem 1. By the corollary to theorem 2 we may assume that $f$ and $g$ preserve Lebesgue measure. Since $f$ and $g$ are ergodic it follows that $h$ preserves Lebesgue measure as well since $h_{*} \lambda$ and $h_{*}^{-1} \lambda$ are absolutely continuous invariant measures for $f$ and $g$ respectively. Countable-to-one locally non-singular maps have Jacobian derivatives (see [3], [4] and [7] for a discussion of these and their properties). We denote these derivatives by $|D|$ as in ordinary differentiation.

$$
|D h|=1 \quad \text { so by the chain rule }|D f| \cdot h=|D g| \text {. }
$$

$|D f|$ is constant a.e. if and only if $|D g|$ is, and in that case there is an integer $n>1$ and two complex numbers of unit modulus $\alpha, \beta$ such that $g(z)=\alpha z^{n}$ and $f(z)=\beta z^{n}$. These two are conjugate by $h(z)=\gamma z$ where $\gamma$ satisfies $\gamma^{n-1}=\alpha / \beta$. So we are reduced to dealing with the case where neither $|D f|$ nor $|D g|$ is constant a.e. This case is achieved by the next proposition which finishes the proof of theorem 1 .

Proposition 1. Let $f$ and $g$ be $C^{2}$ expanding maps of $S^{1}$ which preserve Lebesgue measure. Suppose that $D f$ or $D g$ is not constant and that $f h=h g$ for an absolutely continuous conjugacy $h$. Then there is an isometry $R$ of the circle such that $h=R$ a.e. Proof. $D f$ is not constant. As $D f$ is $C^{1}$ we may find a closed interval $I \subset S^{1}$ such that $|D f|$ is monotone on $I$, maps $I$ to the closed interval $J$ in $\mathbb{R}$ and has a $C^{1}$ inverse $|D f|^{-1}: J \rightarrow I .|D g|^{-1}(J)$ is a denumerable collection of open intervals, so one of these intervals $K$ must intersect $h^{-1}(I)$ in a set of positive measure $X$. So $X \subset K$, $|D g|: K \rightarrow J,|D f|^{-1}: J \rightarrow I$ and $h\left|X=\left(|D f|^{-1}|D g|\right)\right| X$. Now $\psi=|D f|^{-1}|D g|: K \rightarrow I$ is a $C^{\prime}$ map with bounded derivative. Thus $h \mid X$ is Lipschitz.

We will now use the dynamics to spread this Lipschitz property of the conjugacy $h$ on $X$ to a set of measure arbitrarily near 1 . The Lipshitz constant will be uniform so that we will conclude that $h$ agrees a.e. with a Lipschitz map. Then we have a continuous measure preserving conjugacy, that is an isometry between $f$ and $g$ proving the proposition.

Let $c$ work in the distortion lemma for $f$ and $g$ and let $L$ be a Lipschitz constant for the conjugacy $h$ on $X$. Consider a small interval $I$ nearly filled by $X$ which can be expanded by $f^{n}$ injectively to almost fill an interval of length $\frac{1}{3}$. Let $Y=f^{n}(X \cap I)$. Take two points $x, y$ in $Y$ whose distance is $\leq \frac{1}{3}$ and written as $\left(c^{2} L\right)^{-1} \varepsilon$. Let $\bar{x}, \bar{y}$ be the pre-images by $f^{n}$ of $x$ and $y$ in $I$, and let $D=\left|\left(f^{n}\right)^{\prime}(\bar{x})\right|$. The distance between $\bar{x}$ and $\bar{y}$ is at most $\left(c D^{-1}\right)\left(c^{2} L\right)^{-1} \varepsilon=(c D)^{-1}(L)^{-1} \varepsilon$ by the distortion lemma. Thus the distance between $h \bar{x}$ and $h \bar{y}$ is at most $(c D)^{-1} \varepsilon$. The derivative of $g^{n}$ at $h \bar{x}$ is also $D$. Thus by corollary 2 the distance between $g^{n}(h \bar{x})$ and $g^{n}(h \bar{y})$ is at most $\varepsilon$. 
But $g^{n}(h \bar{x})=h f^{n} \bar{x}=h(x)$ and $g^{n}(h \bar{y})=h f^{n}(\bar{y})=h(y)$. So the distance between $h(x)$ and $h(y)$ is at most $\left(c^{2} L\right)$ times the distance between $x$ and $y$ (as long as this distance as measured in $f^{n}(I)$ is $\left.\leq\left(c^{2} L\right)^{-1}\right)$.

Proceeding in this way we can find a sequence of sets $Y_{n}$ contained in intervals $I_{n}$ so that $I_{n} \rightarrow I$ an interval of length $\frac{1}{3}$ say and measure $Y_{n} \rightarrow \frac{1}{3}$. Then the conjugacy is Lipschitz on $I$.

Now an a.e. conjugacy which locally agrees with a continuous map on some open set locally agrees with a continuous map everywhere (by spreading again). Thus we are done.

Remark. Theorem 1 requires the orientation hypothesis since $z^{n}$ and $z^{-n}$ are absolutely continuously conjugate but are not conjugate by a homeomorphism.

The existence of an absolutely continuous conjugacy implies the isomorphism of the Jacobian derivative namely, $|D f| \cdot h=|D g|$ where $h$ is a measure preserving isomorphism of the circle. In the real analytic case the isomorphism of the Jacobians themselves almost determines an isometric conjugacy.

THEOREM 3. Let $f$ and $g$ be real analytic expanding endomorphisms of $S^{1}$ which preserve Lebesgue measure. Suppose that the Jacobian derivatives of $f$ and $g$ are isomorphic, i.e. that there exists a measure-preserving isomorphism of $S^{1}, h: S^{1} \rightarrow S^{1}$ such that $|D f| \cdot h=|D g|$. Then there are isometries $R_{1}$ and $R_{2}$ of $S^{1}$ such that $R_{1} f R_{1}^{-1}=R_{2} g$.

Proof. If $|D f|$ and $|D g|$ are a.e. constant then there is an integer $n$ and complex numbers $\alpha, \beta$ of modulus one such that $f$ and $g$ are $\alpha z^{ \pm n}$ and $\beta z^{ \pm n}$ and the conclusion of the theorem is clear. So we may assume that $|D f|$ is not locally constant. Then construct $\psi, K, J, I$ and $X$ as in proposition 1 with the additional provision that $|D f|^{-1},|D g|$ and $\psi$ are real analytic, $\psi=|D f|^{-1}|D g|$ and $\psi|X=h| X$. Differentiating the last equation at the density points of $X$ gives $|D \psi|=|D h|=1$ and by analytic continuation $|D \psi|=1$ on $K$ and $\psi$ is the restriction of an isometry $R_{1}$ to $K$. $\left|D\left(R_{1}^{-1} f R_{1}\right)\right|=|D f| \cdot R_{1}$ so $\left|D\left(R_{1}^{-1} f R_{1}\right)\right|=|D g|$ on $X$.

Since $R_{1}^{-1} f R_{1}$ and $g$ are real analytic, analytic continuation shows that $D\left(R_{1}^{-1} f R_{1}\right)= \pm D g$ on all of $S^{1}$ and there is an isometry $R_{2}$ of $S^{1}$ such that $R_{1}^{-1} f R_{1}=R_{2} \mathrm{~g}$.

Open problem. In other words theorem 3 says that $f$ and $g$ are conjugate up to a phase factor if their Jacobians are isomorphic. If $f$ and $g$ are absolutely continuously conjugate they are real analytically conjugate by theorem 1 . Thus the phase factor is a measure theoretic conjugacy invariant. In summary the Jacobian invariant and a phase factor form a complete system of measure theoretical (and thus real analytical) conjugacy invariants for real analytic expanding maps.

It would be useful to have a measure theoretical definition of a phase factor invariant for expanding endomorphism of the circle. This is the promised problem.

\section{Section 2. Finite Blaschke products}

A finite Blaschke product

$$
B: z \rightarrow a_{0} \prod_{i=0}^{n}\left(z-\bar{a}_{i}\right) /\left(1-a_{i} z\right) \quad n>1,\left|a_{0}\right|=1,\left|a_{i}\right|<1 \quad \text { for } i>0
$$


determines an expanding endomorphism of the circle $\{z:|z|=1\}$ iff each fixed point $u \in S$ is expanding. The necessity is obvious. The sufficiency is explained in the proof of:

THEOREM 4. If two expanding Blaschke products are absolutely continuously conjugate they are conjugate by a Möbius transformation of the circle.

Proof. The degree of $B$ on the circle is $n$ so the total Lefschetz number of fixed points has absolute value $n-1$. If each fixed point on $S^{1}$ is expanding there must be $(n-1)$ of them. The degree of the Blaschke product as a rational map of $S^{2}$ is also $n$ and the total Lefschetz number of fixed points there is $n+1$.

Thus in the case of $(n-1)$ fixed points on $S^{1}$ which are expanding there must be 2 other fixed points in $S^{2}$ off of $S^{1}$ (and symmetric by the inversion in $S^{1}$ ).

If $z_{0}$ is the fixed point inside the disk, then $B\left(z_{0}\right)=z_{0}$ implies $B$ on $S^{1}$ preserves the Poisson measure $\mu\left(z_{0}\right)$ on the circle relative to $z_{0}$ (this is well known and follows easily since $B$ is analytic, preserves the unit disk, preserves the class of bounded harmonic functions $h$ on the disk, and these satisfy

$$
\left.h(z)=\int_{S^{1}}(\text { boundary values of } h) d \text { (Poisson relative to } z\right) ;
$$

see [2] and the references therein for this argument).

We may transform $\mu\left(z_{0}\right)$ to Lebesgue measure by the Möbius transform $t$ carrying $z_{0}$ to the centre of the disk. Call $t B t^{-1}$ the normalized Blaschke product associated to $B$. Since $t B t^{-1}$ preserves Lebesgue measure and is $n$-to- 1 it is expanding in the standard metric on $S^{1}$.

Thus if two expanding Blaschke products are absolutely continuously conjugate their normalized versions are conjugate by an isometry of the circle using proposition 1. This proves the theorem 4.

Both authors were partially supported by NSF grant no. MCS-8201267.

\section{REFERENCES}

[1] K. Krzyzewsky. Some results on expanding mappings. Asterisque 50 (1977), 205-218.

[2] N. F. G. Martin. On finite Blaschke products whose restriction to the unit circle are exact endomorphisms. Bull. London Math. Soc. 15 (1983), 343-348.

[3] W. Parry. Entropy and Generators in Ergodic Theory. Benjamin, 1969.

[4] W. Parry \& P. Walters. Endomorphisms of a Lebesgue space. Bull. Amer. Math. Soc. (1972), 272-276.

[5] R. Sacksteder. The measures invariant under an expanding map. In Geometrie Differentielle, Springer Lecture Notes, 392, Springer-Verlag: Berlin; pp. 179-194.

[6] M. Shub. Endomorphisms of compact differentiable manifolds. Amer. J. Math XCI (1969), 175-199.

[7] P. Walters. Some results on the classification of measure preserving transformations. In Recent Advances in Topological Dynamics. Lecture Notes in Math. Vol. 319, pp. 255-276. Springer-Verlag, 1973.

[8] P. Walters. Invariant measures and equilibrium states for some mappings which expand distances. Trans. Amer. Math. Soc. 236 (1978), 121-153. 